\title{
Comentario al nuevo documento de consenso de la Sociedad Europea de Hipertensión 2021: "Guía práctica sobre la medida de la presión arterial en consulta y ambulatoria"
}

\author{
Eugenia García-Zaragoza'ำ, Miguel Ángel María-Tablado² \\ 1. Farmacéutica comunitaria. Alicante. 2. Médico de Familia. Madrid.
}

\section{PALABRAS CLAVE}

Hipertensión, diagnóstico, presión arterial, farmacias

\section{ABREVIATURAS}

AMPA: automedida ambulatoria de presión arterial

ESH: Sociedad Europea de

Hipertensión

HBB: hipertensión de "bata blanca"

HE: hipertensión enmascarada

HR: hipertensión resistente

HTA: hipertensión

MAPA: monitorización ambulatoria

de presión arterial

PA: presión arterial

$\mathrm{RCV}$ : riesgo cardiovascular

\section{KEYWORDS}

Hypertension, diagnosis, blood pressure, pharmacies

\section{RESUMEN}

En el presente trabajo se comentan los aspectos más relevantes y novedosos de la nueva Guía de la Sociedad Europea de Hipertensión (ESH) 2021. De ellos el más significativo es la inclusión como método de medida de la presión arterial la medida en Farmacia Comunitaria y su comparación frente al resto de métodos existentes (en consulta, MAPA y AMPA). A pesar de la falta de estudios al respecto, posee un enorme potencial y se postula como un método muy adecuado para el cribado de la hipertensión arterial (HTA) y adecuado para el seguimiento y diagnóstico de los pacientes con hipertensión.

Commentary to the new consensus document of the European society of Hypertension 2021: "Practice guidelines for office and out-of-office blood pressure measurement"

\section{ABSTRACT}

This paper discusses the most relevant and novel aspects of the new European Society of Hypertension (ESH) practice guidelines 2021. Of these, the most significant is the inclusion of the measurement in Community Pharmacies as a method of blood pressure (BP) measurement and its comparison against the other existing methods (office, ambulatory and home BP measurements). Despite the weak evidences, it has enormous potential and is postulated as very useful method for screenning of hypertension and useful for follow-up and diagnosis of patients with hypertension.

\section{INTRODUCCIÓN}

La hipertensión (HTA) es factor de riesgo de morbimortalidad en todo el mundo y su diagnóstico se basa exclusivamente en la precisión de la medida de la presión arterial (PA). Un método inexacto de diagnostico puede catalogar al paciente en hipertenso o lo contrario, con el significado clínico de ello.

Actualmente en nuestro medio existen diferentes métodos para realizar la medida adecuada: unos en la consulta generalmente de Atención Primaria (auscultatorio, automático o no observado), otros en el domicilio (Monitorización ambulatoria de la presión arterial, MAPA; Automedida de la presión arterial, AMPA) y, por último, fuera de consulta y domicilio en las farmacias comunitarias.

La mayoría de guías clínicas recomiendan el empleo de AMPA y MAPA para el diagnóstico de la Hipertensión de "bata blanca" (HBB), Hipertensión enmascarada (HE) e Hipertensión pseudorresistente y resistente (HR). Sin embargo, de momento, la toma de decisiones respecto a la clasificación, umbral y objetivo de tratamiento se basa en la medida de la PA en consulta.

Financiación: ninguna

Conflicto de intereses: ninguno.

Cite este artículo como: García-Zaragoza E, María-Tablado MÁ. Comentario al nuevo documento de consenso de la Sociedad Europea de Hipertensión 2021: "Guia práctica sobre la medida de la presión arterial en consulta y ambulatoria". Farmacéuticos Comunitarios. 2021 0ct 06; 13 (4): 21-27. doi:10.33620/FC.2173-9218.(2021/Nol13).004.04

Correspondencia: Eugenia Garcia-Zaragoza (eugenia.garcia@uv.es).

ISSN 1885-8619 @SEFAC (Sociedad Española de Farmacia Clínica, Familiar y Comunitaria). Todos los derechos reservados. 
El presente documento tiene como objetivo realizar un resumen del documento de consenso sobre recomendaciones clave en la toma de la tensión arterial, recientemente elaborado por la ESH (Sociedad Europea de Hipertensión); en concreto por el grupo de trabajo de Monitorización y Variabilidad de la PA (1).

La principal novedad que aporta esta guía es la inclusión de las mediciones llevadas a cabo en Farmacia Comunitaria, su procedimiento, ventajas e inconvenientes frente al resto de métodos de medida. Si bien parecen tener un enorme potencial, necesitan más estudios que las validen frente a los de métodos usados tradicionalmente. Asimismo, menciona otros métodos más innovadores que actualmente están al alcance de los pacientes como son las tecnologías móviles y dispositivos tipo bandas de muñeca que, a pesar de estar aún por desarrollar y validar, es importante que los profesionales sanitarios conozcamos a futuro.

\section{ASPECTOS COMUNES A LOS DISTINTOS MÉTODOS DE MEDIDA}

A lo largo de toda la guía se hace especial hincapié en la importancia de la precisión y reproducibilidad de las medidas de la PA. Desde 2018 existe un protocolo internacional de validación de aparatos electrónicos de medida de la PA (AAMI) ESH/ISO). Sólo se deberían utilizar los aparatos validados según dicho protocolo, aunque la realidad es que de los casi 4.000 aparatos disponibles a nivel mundial, solo el $10 \%$ está validado. En España actualmente existe el listado de aparatos validados de SEH-LELHA https://seh-lelha.org/aparatos-pa/

Siempre hay que comprobar que el dispositivo a utilizar esté validado en la población con la que se esté trabajando, ya que los monitores que han sido validados en adultos pueden no serlo en otras poblaciones: niños, embarazadas, diámetro de brazo $>42 \mathrm{~cm}$, pacientes con arritmias (fibrilación auricular).

Se prefieren los dispositivos automáticos a los auscultatorios. Pero siempre debe estar validado y calibrado de forma periódica (anual).

Otro punto clave en la medida de la PA es el tamaño del manguito, ya que si es inadecuado puede dar una lectura errónea. Los pacientes con diámetro del brazo $>42 \mathrm{~cm}$ deberían utilizar manguitos de forma cónica o en su defecto tensiómetros de muñeca. Los dispositivos automáticos suelen incorporar manguitos de distintos tamaños y la elección del diámetro es fundamental. Otras veces llevan un manguito que cubre un amplio rango de tallas y se acopla a la mayoría de brazos de adulto.

En la tabla 1 recordamos los fenómenos de Hipertensión de "bata blanca" (HBB) y enmascarada (HE), su frecuencia y actitud ante ellos.

\section{MEDICIÓN EN CONSULTA}

Sigue siendo la forma mas utilizada y en muchas ocasiones la única. Es el método más estudiado y utilizado en ensayos de los que se deduce la clasificación HTA, umbrales y objetivos de control. Nos puede producir una falsa sensación de seguridad y por ello siempre que sea posible se debería confirmar con MAPA o AMPA. En la tabla 2 se recoge el procedimiento correcto para su realización.

\section{Diagnóstico de HTA}

La guía recomienda realizarlo al menos tras 2-3 visitas a la consulta (dependiendo del nivel de PA y RCV). Nunca hacer diagnóstico en una sola consulta salvo cifras $>180 / 110 \mathrm{mmHg}$, evidencia de lesión en órganos diana (corazón, riñón, retina, cerebro) o RCV alto. En la primera visita se debería medir la PA

Tabla 1 Diagnóstico y manejo de la Hipertensión de bata blanca y enmascarada

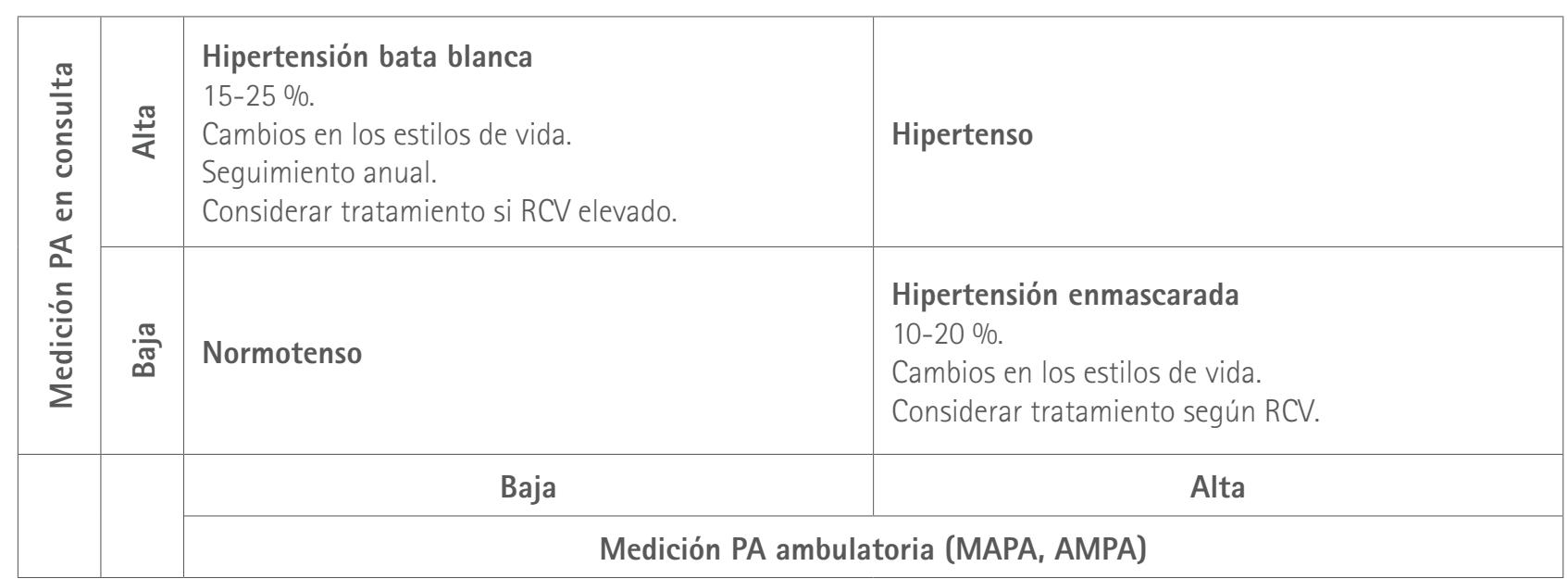

$\mathrm{RCV}$ : riesgo cardiovascular; PA: presión arterial. 
Tabla 2 Medición en consulta: método, ventajas e inconvenientes

\begin{tabular}{|c|c|c|c|c|}
\hline Condiciones de medida & Postura de medida & Medidas & Ventajas & Limitaciones \\
\hline $\begin{array}{l}\text { Ambiente tranquilo } \\
\text { y temperatura } \\
\text { agradable. } \\
\text { No tabaco, café, } \\
\text { comida o ejercicio } \\
30 \text { min antes. } \\
\text { Sentado y relajado } \\
5 \text { min antes. } \\
\text { No hablar. } \\
\text { Aparato validado y } \\
\text { calibrado (anual). } \\
\text { Manguito adecuado. }\end{array}$ & $\begin{array}{l}\text { Sentado y espalda } \\
\text { apoyada. } \\
\text { Piernas descruzadas y } \\
\text { pies apoyados. } \\
\text { Antebrazo } \\
\text { descubierto, a la } \\
\text { altura del corazón y } \\
\text { apoyado sobre una } \\
\text { mesa. }\end{array}$ & $\begin{array}{l}\text { Tomar al menos } \\
3 \text { tomas ( } 2 \text { si son } \\
\text { normales) con } \\
1 \text { min de diferencia } \\
\text { entre ellas. } \\
\text { Utilizar el promedio. }\end{array}$ & $\begin{array}{l}\text { Elevada } \\
\text { disponibilidad. } \\
\text { Utilizado en la } \\
\text { mayoría de ensayos: } \\
\text { evidencia alta de } \\
\text { vinculación de datos } \\
\text { de PA en consulta y } \\
\text { RCV. }\end{array}$ & $\begin{array}{l}\text { Sobreestimación de } \\
\text { la HTA. } \\
\text { Baja reproducibilidad } \\
\text { de una sola visita, } \\
\text { que proporciona } \\
\text { diagnósticos poco } \\
\text { precisos. } \\
\text { Favorece HBB. } \\
\text { No detecta HE. }\end{array}$ \\
\hline
\end{tabular}

RCV: riesgo cardiovascular; HTA: hipertensión; HBB: hipertensión de bata blanca; HE: hipertensión enmascarada.

en ambos brazos. Si existe diferencia se debe repetir y tomar la cifra más alta.

Puede ser necesario la medida en bipedestación si hay sospecha de hipotensión postural, en particular ancianos, Enfermedad de Parkinson o deterioro cognitivo.

\section{MONITORIZACIÓN AMBULATORIA DE PRESIÓN ARTERIAL (MAPA)}

Proporciona múltiples lecturas de PA en el entorno habitual del paciente durante sus actividades diarias, incluido el descanso nocturno. Diagnostica HBB y HE. Está recomendado en varias guías como mejor método de diagnóstico. En las tablas 3 y 4 se resumen las características, indicaciones y requerimientos para su realización.

\section{Interpretación de la MAPA: valores de corte} Diagnóstico de HTA: media 24 horas $>130 / 80 \mathrm{mmHg}$, Hipertensión diurna $>135 / 85 \mathrm{mmHg}$, Hipertensión nocturna $>120 / 70 \mathrm{mmHg}$.

Patrón Dipper $>10$ \% disminución noche/día, Patrón No dipper $<10 \%$ disminución.

Tabla 3 Características e indicaciones de la MAPA

\begin{tabular}{|c|c|c|c|c|}
\hline Ventajas & Limitaciones & \multicolumn{3}{|l|}{ Indicaciones } \\
\hline Medición 24 h. & Baja disponibilidad. & Diagnóstico inicial & HTA en tratamiento & Cuándo repetir \\
\hline $\begin{array}{l}\text { Actividades diarias. } \\
\text { Detecta HBB, HE y } \\
\text { HR. } \\
\text { Detecta HTA } \\
\text { nocturna y patrón } \\
\text { no dipper. } \\
\text { Detecta } \\
\text { sobretratamiento. }\end{array}$ & $\begin{array}{l}\text { Método molesto } \\
\text { para el paciente. } \\
\text { Puede causar } \\
\text { rechazo. } \\
\text { Baja } \\
\text { reproducibilidad. } \\
\text { Dificultad en } \\
\text { registrar el sueño } \\
\text { real. }\end{array}$ & $\begin{array}{l}\text { Diagnóstico de } \\
\text { HTA. } \\
\text { Detección de: } \\
\text { - HBB. } \\
\text { - HE. } \\
\text { - HTA nocturna. } \\
\text { - Patrón no dipper. }\end{array}$ & $\begin{array}{l}\text { Detección de: } \\
\text { - HBB. } \\
\text { - HE. } \\
\text { - HR. } \\
\text { - Mal controlada. } \\
\text { - Hipotensión } \\
\text { sintomática. } \\
\text { - HTA nocturna. } \\
\text { - Patrón no dipper. } \\
\text { Desacuerdo AMPA/ } \\
\text { consulta. }\end{array}$ & $\begin{array}{l}\text { Para asegurar buen control } \\
\text { sobre todo si RCV alto. } \\
\text { Anual cuando HTA controlada. } \\
\text { Cada } 3 \text { meses en falta de } \\
\text { control. }\end{array}$ \\
\hline
\end{tabular}

HTA: hipertensión; HBB: hipertensión de bata blanca; HE: hipertensión enmascarada; HR: hipertensión resistente; RCV: riesgo cardiovascular. 
Tabla 4 Realización de la MAPA

\begin{tabular}{|c|c|c|c|}
\hline Requerimientos & locación & etirada & Instrucciones a pacientes \\
\hline $\begin{array}{l}\text { Escoger un dia de } \\
\text { trabajo rutinario del } \\
\text { paciente. } \\
\text { Necesita } 10-15 \text { min } \\
\text { de instalación sobre el } \\
\text { paciente. } \\
\text { Aparato validado y } \\
\text { calibrado (anual). } \\
\text { Manguito adecuado. }\end{array}$ & $\begin{array}{l}\text { Medidas cada } 20-30 \text { min } \\
\text { (dia y noche). } \\
\text { Manguito en brazo no } \\
\text { dominante y ajustado al } \\
\text { diámetro. } \\
\text { Hacer primera toma de } \\
\text { PA de prueba. }\end{array}$ & $\begin{array}{l}\text { Retirar tras } 24 \mathrm{~h} . \\
\text { No válida si }<20 \\
\text { medidas correctas } \\
\text { diurnas } 0<7 \\
\text { nocturnas. } \\
\text { Determinar los } \\
\text { períodos diurno y } \\
\text { nocturno sólo en } \\
\text { base al documento de } \\
\text { registro del paciente. }\end{array}$ & $\begin{array}{l}\text { Realizar las actividades diarias habituales. } \\
\text { Durante la medida permanecer en } \\
\text { silencio y con el brazo relajado. } \\
\text { No ducharse ni bañarse con la MAPA. } \\
\text { Enseñar el manejo del aparato para que } \\
\text { lo pueda apagar o recolocar él mismo. } \\
\text { Proporcionar un documento de registro } \\
\text { de tomas de medicación, horarios de } \\
\text { sueño o problemas. }\end{array}$ \\
\hline
\end{tabular}

PA: presión arterial.

\section{AUTOMEDIDA AMBULATORIA DE PRESIÓN ARTERIAL (AMPA)}

Muy utilizado. Proporciona múltiples medidas en entorno habitual del paciente. Identifica HBB y HE. Recomendado como mejor método de seguimiento a largo plazo.

Lo ideal sería un dispositivo con conexión a móvil, PC o internet para exportar los datos. Los dispositivos de muñeca son menos recomendables por su baja sensibilidad salvo en pacientes con brazos muy anchos. Los dispositivos de dedos, bandas de muñeca... no son recomendables. Sus caracteristicas, indicaciones y requerimientos para su realización se resumen en las tablas 5 y 6 .

\section{Interpretación de AMPA: valores de corte}

Descartar las medidas del primer día y realizar la media del resto de medidas. Las medidas aisladas no tienen valor.

Diagnóstico de HTA: media AMPA >135/85 mmHg.

\section{MEDICIÓN EN FARMACIA COMUNITARIA}

Su aparición como método de medida "oficial" de la PA en una guia europea sobre el manejo de la HTA es la gran novedad que justifica nuestro interés y el presente resumen. En nuestro pais (y en muchos otros de Europa), la medida protocolizada de la PA es una práctica cada vez más habitual en las farmacias, pero debido al reducido número de estudios publicados al respecto, su manejo y potencial no están del todo establecidos. Por la misma razón tampoco se dispone de una evidencia científica sólida para establecer los valores umbral de diagnóstico de HTA en este entorno.

Se prefieren los dispositivos automáticos que realizan una triple toma. La toma de la PA se debe realizar en una zona tranquila de la farmacia. La tabla 7 resume sus ventajas y limitaciones.

Tabla 5 Caracteristicas e indicaciones de AMPA

\begin{tabular}{|l|l|l|l|}
\hline Ventajas & Limitaciones & Indicaciones \\
\hline Bajo coste. & Dispositivos no validados. & Diagnóstico inicial & HTA en tratamiento \\
\cline { 2 - 4 } Elevada disponibilidad. & $\begin{array}{l}\text { Tamaño manguito } \\
\text { inadecuado. }\end{array}$ & Confirmar diagnóstico de & $\begin{array}{l}\text { Ideal para seguimiento } \\
\text { HTA. }\end{array}$ \\
Ideal para seguimiento. & Técnica de determinación no & Detección de HBB y HE. & $\begin{array}{l}\text { pacientes a largo plazo. } \\
\text { Detecta HBB y HE. }\end{array}$ \\
Confirma HR y mal & estandarizada. & & Identifica HBB y HE. \\
controlada. & Ansiedad en el paciente. & & Asegura un control estricto \\
Detecta hipotensión. & Riesgo de cambios de & & Mejora de la adherencia. \\
Mejora adherencia. & tratamiento por el paciente. & & \\
Puede usarse con & Datos falseados por & & \\
telemonitorización. & paciente. & & \\
& Infraestimación por & & \\
\end{tabular}

HTA: hipertensión; HBB: hipertensión de bata blanca; HE: hipertensión enmascarada; HR: hipertensión resistente. 
Tabla 6 Realización y manejo de AMPA

\begin{tabular}{|c|c|c|c|}
\hline Procedimiento & Formación del paciente & Programación & \\
\hline \multirow{2}{*}{$\begin{array}{l}\text { Condiciones y postura } \\
\text { de medida similares a } \\
\text { medición en consulta } \\
\text { (ver tabla 2). }\end{array}$} & \multirow[b]{2}{*}{$\begin{array}{l}\text { Dispositivo correcto. } \\
\text { Condiciones y postura } \\
\text { adecuados. } \\
\text { Programar mediciones: } \\
\text { - antes de consulta } \\
\text { - entre visitas. } \\
\text { Interpretación de resultados: } \\
\text { Variabilidad de la PA. } \\
\text { Informar que hacer: } \\
\text { - si PA muy alta } \\
\text { - si PA muy baja. }\end{array}$} & $\begin{array}{l}\text { Antes de consulta o para el } \\
\text { diagnóstico }\end{array}$ & $\begin{array}{l}\text { Seguimiento a largo plazo del } \\
\text { tratamiento HTA }\end{array}$ \\
\hline & & $\begin{array}{l}\text { Mediciones durante } 7 \text { dias } \\
\text { (mínimo } 3 \text { días). } \\
\text { Matutinas y vespertinas. } \\
\text { Antes de fármacos y de } \\
\text { comidas. } \\
\text { Dos mediciones con } 1 \mathrm{~min} \\
\text { de diferencia. }\end{array}$ & $\begin{array}{l}\text { Realizar mediciones } \\
\text { duplicadas } 1 \text { o } 2 \text { por semana, } \\
\text { mínimo } 2 \text { por mes. }\end{array}$ \\
\hline
\end{tabular}

HTA: hipertensión; PA: presión arterial.

Tabla 7 Realización y manejo de la medición en Farmacia Comunitaria

\begin{tabular}{|l|l|l|}
\hline Ventajas & Limitaciones & Procedimiento \\
\hline Accesible y sin cita. & $\begin{array}{l}\text { Dispositivos no validados; tamaño } \\
\text { manguito inadecuado; técnica de } \\
\text { Útil en el diagnóstico y seguimiento. } \\
\text { Disminución consultas médico de } \\
\text { familia y costes. }\end{array}$ & $\begin{array}{l}\text { Condiciones y postura de medida } \\
\text { similares a medición en consulta } \\
\text { (ver tabla 2). }\end{array}$ \\
No implica HBB (o menos). & $\begin{array}{l}\text { No conoce cuál es la cifra } \\
\text { diagnóstica en este medio. } \\
\text { Alternativa AMPA o MAPA. }\end{array}$ & \\
& $\begin{array}{l}\text { Puede aumentar las derivaciones al } \\
\text { médico de familia*. }\end{array}$ & \\
\hline
\end{tabular}

* Siempre que la realización de la medida sea correcta, un incremento en las derivaciones supone una ventaja al mejorar el control y abordaje multidisciplinar del paciente.

\section{Interpretación de la medición en Farmacia Comunitaria: valores de corte}

Si la media de 2-3 medidas es $>135 / 85 \mathrm{mmHg}$ sugiere HTA no controlada. El diagnóstico y tratamiento no debe basarse únicamente en esta técnica.

En algunas farmacias y otros espacios públicos, la toma de la PA se realiza en lugar de por el método anterior mediante unos quioscos de PA o dispositivos automáticos que activa el propio paciente al insertar una moneda. El aparato realiza automáticamente 2-3 lecturas y emite un ticket con los valores. Están muy poco estudiados, por lo que se desconocen las cifras umbral de diagnóstico de HTA. A pesar de ser un método accesible y útil para el screening, el aparato puede no estar validado, el manguito puede no ser el adecuado y frecuentemente no hay seguimiento por parte de profesionales sanitarios.

\section{RESUMEN}

A modo de resumen de lo leído presentamos las tablas 8 y 9 con ventajas, limitaciones, umbral diagnóstico y utilidad de todos los métodos de medida de la PA. 
Tabla 8 Comparativa sobre las ventajas e inconvenientes de cada uno de los métodos de medida de la PA y su utilidad en el diagnóstico de HTA

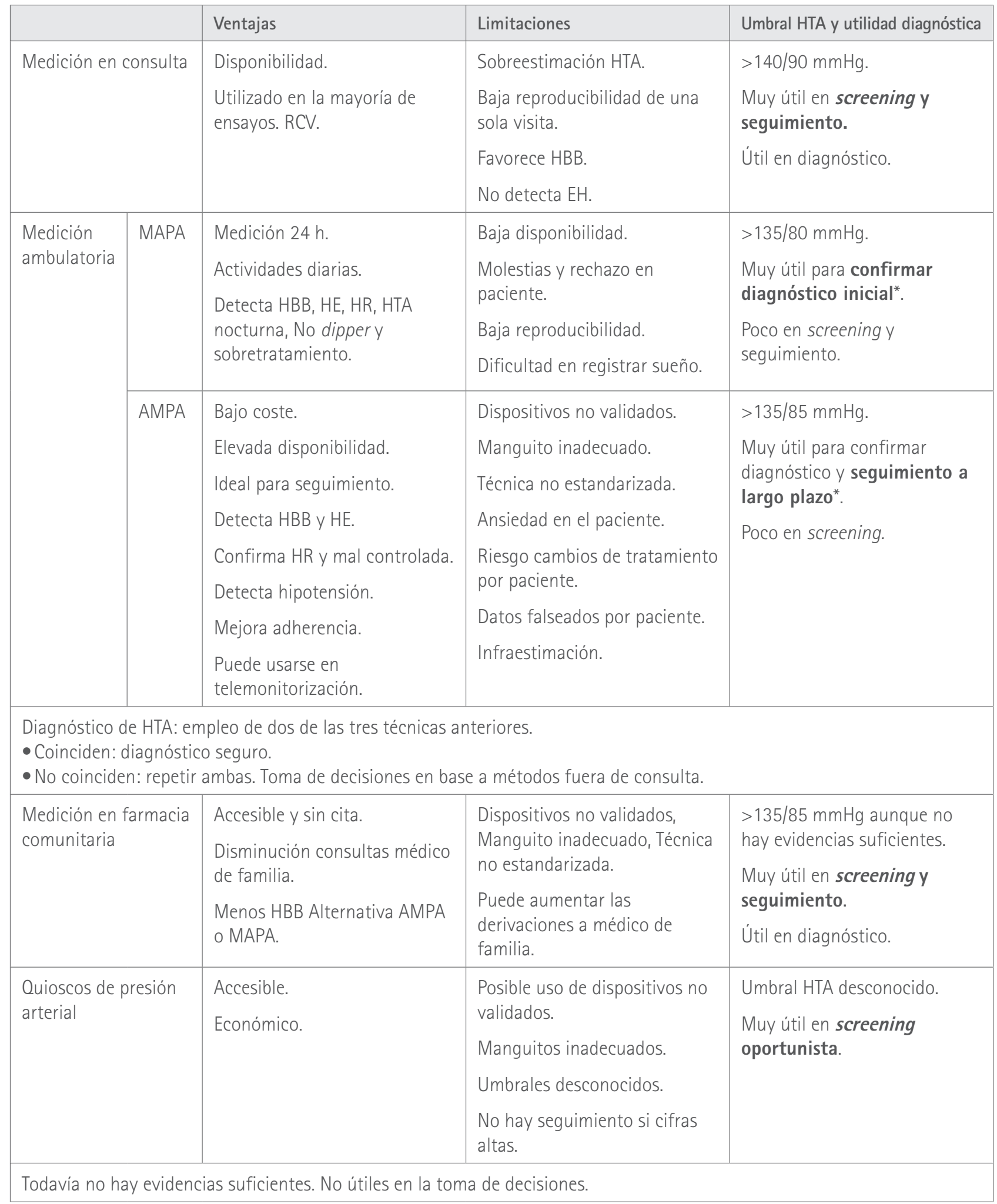

En negrita su indicación principal.

* Método preferido.

RCV: riesgo cardiovascular; HTA: hipertensión; HBB: hipertensión de bata blanca; HE: hipertensión enmascarada; HR: hipertensión resistente. 
Tabla 9 Utilidad en la práctica clínica de cada uno de los métodos de medición de la presión arterial

\begin{tabular}{|l|l|l|l|l|l|}
\hline \multirow{2}{*}{} & \multirow{2}{*}{$\begin{array}{l}\text { Medición en } \\
\text { consulta }\end{array}$} & \multicolumn{2}{|l|}{ Medición ambulatoria } & $\begin{array}{l}\text { Medición } \\
\text { farmacia } \\
\text { comunitaria }\end{array}$ & $\begin{array}{l}\text { Quioscos de } \\
\text { presión arterial }\end{array}$ \\
\cline { 3 - 6 } Screening & MAPA & AMPA & & \\
\hline Confirmación diagnóstico & & & & & \\
\hline Seguimiento & & & & & \\
\hline
\end{tabular}

: utilidad principal; $\odot$ : utilidad media;

baja utilidad.

\section{REFERENCIAS BIBLIOGRÁFICAS}

1. Stergiou GS, Palatini P, Parati G, et al. 2021 European Society of Hypertension practice guidelines for office and out-of-office blood pressure measurement. Journal of Hypertension. $2021 \mathrm{Jul} ; 39$ (7):1293-1302. doi:10.1097/HJH.0000000000002843. 